\title{
MicroRNA-671-3p regulates the development of knee osteoarthritis by targeting TRAF3 in chondrocytes
}

\author{
ZHENGJIE LIU $^{1 *}$, SHUNGUANG CHEN ${ }^{1 *}$, YEZI YANG ${ }^{2}$, \\ SHENGJUN LU ${ }^{1}$, XUNMING ZHAO ${ }^{1}$, BIAO HU ${ }^{1}$ and $\mathrm{HONG} \mathrm{PEI}^{1}$
}

Departments of ${ }^{1}$ Orthopedics and ${ }^{2}$ Clinical Laboratory, Jingzhou Central Hospital, Jingzhou, Hubei 434020, P.R. China

Received April 28, 2018; Accepted November 27, 2018

DOI: $10.3892 / \mathrm{mmr} .2019 .10488$

\begin{abstract}
Osteoarthritis (OA) is a degenerative joint disease characterized by articular cartilage degradation and joint inflammation. A previous study showed that microRNA (miR)-671-3p is involved in the development of OA, however, its function and molecular target in chondrocytes during the pathogenesis of OA remain to be fully elucidated. In the present study, miR-671-3p was significantly downregulated in knee OA cartilage tissues compared with normal cartilage tissues. The expression levels of pro-inflammatory cytokines, including interleukin (IL)-1 $\beta$, IL-6, IL-8 and tumor necrosis factor (TNF)- $\alpha$, in the knee OA cartilage tissues were significantly higher than those in the normal cartilage tissues. Through gain-of-function and loss-of-function experiments, miR-671-3p was shown to significantly affect matrix synthesis gene expression, cell proliferation, apoptosis and inflammation in chondrocytes from patients with OA. Subsequent bioinformatics analysis identified potential target sites of the miR-671-3p located in the 3'untranslated region of TNF receptor-associated factor (TRAF3). The results of a dual-luciferase reporter assay showed that TRAF3 is a target gene of miR-671-3p. Western blot analysis demonstrated that miR-671-3p inhibited the gene expression of TRAF3. Furthermore, the restoration of TRAF3 markedly abrogated the effect of miR-671-3p. Taken together, the present study suggests that miR-671-3p may be important in the pathogenesis of OA through targeting TRAF3 and regulating chondrocyte apoptosis and inflammation, which may be a potential molecular target for OA treatment.
\end{abstract}

Correspondence to: Dr Hong Pei, Department of Orthopedics, Jingzhou Central Hospital, 60 Jingzhong Road, Jingzhou, Hubei 434020, P.R. China

E-mail: pei_hong0105@126.com

*Contributed equally

Key words: osteoarthritis, microRNA-671-3p, chondrocyte apoptosis, inflammation, TRAF3

\section{Introduction}

Osteoarthritis (OA) is a leading cause of disability and is characterized by articular cartilage destruction, subchondral bone changes and synovial tissue inflammation (1). In previous decades, the incidence of OA has increased substantially in several countries and regions, including the Nordic region (2) and the United States (3). Emerging evidence has revealed that $\mathrm{OA}$ is an inflammatory disease and the increased secretion of various pro-inflammatory chemokines is crucial in the pathogenesis of OA (4). In addition, signaling factors involved in the proliferation, differentiation, senescence and apoptosis of chondrocytes, which is the only cell type in mature cartilage, have been shown to be partially responsible for OA processes (5-7). However, the molecular mechanisms contributing to the pathology and pathophysiology of OA remain to be fully elucidated.

MicroRNAs (miRNAs) constitute a large family of small non-coding RNAs ( 22 nucleotides) that function as post-transcriptional regulators of gene expression by recognizing and binding to partially complementary sites in the 3'-untranslated region (3'-UTR) of target genes for either the cleavage or depression of translation in eukaryotic organisms (8). A number of miRNAs have been implicated in regulating a wide range of complex biological processes, including development, proliferation, differentiation, apoptosis, and immune and inflammation responses (9). The aberrant or deregulated expression of miRNAs is often associated with the biology and pathology of human cancer (10), cardiovascular disease (11) and OA (12), and this is a major target for diagnostic, prognostic and therapeutic interventions. Nugent first examined the differences in the levels of 157 miRNAs between human cartilage and bone in post-mortem OA specimens from donors with no previous joint pain (13). It was found that 30 miRNAs were significantly dysregulated in the cartilage and bone of diseased tissue compared with the normal control (13). Alterations in miRNA expression levels, including miRNA (miR)-9, miR-140, miR-27b and miR-34a, are associated with OA processes (14-17). miR-671-3p is known to be downregulated in the serum of patients with OA, and it has been suggested to be involved in metabolic processes that contribute to OA pathology (18). However, the biological function and the underlying molecular mechanisms of miR-671-3p in OA remain to be elucidated. Pathogen-associated molecular pattern-activated 
Toll-like receptor (TLR) is important in innate immune responses, cell apoptosis and the production of interferons and pro- and anti-inflammatory cytokines (19). As an indispensable component of signaling pathways triggered by members of the tumor necrosis factor (TNF) receptor (TNFR) and TLR families, TNFR-associated factor (TRAF) has been shown to function as a negative regulator of nuclear factor $-\kappa \mathrm{B}$ and mitogen-activated protein kinases $(19,20)$. TRAF-interacting protein is important in regulating inflammatory process in rheumatoid arthritis-proliferative fibroblast-like synoviocytes and is considered a potential therapeutic target for human rheumatoid arthritis (21). Therefore, TRAF may be crucial in the development of OA.

In the present study, the expression of miR-671-3p was detected in human knee OA tissues and normal cartilage tissues, and in OA chondrocytes and normal chondrocytes. This in combination with experiments involving the gain- and loss-of-function of miR-671-3p in OA chondrocytes and a luciferase reporter assay, was used to evaluate the implication of miR-671-3p and its association with TRAF3 in the progression of OA. The results will clarify the potential correlations between miR-671-3p/TRAF3 and the development of OA, which may be conductive to exploitation of a novel strategy for the treatment of OA.

\section{Materials and methods}

Tissue samples and chondrocyte culture. The human knee OA cartilage samples were collected from patients $(n=15$; six women and nine men, aged 50-79 years) undergoing knee replacement surgery at Jingzhou Central Hospital (Jingzhou, China; Table I). None of the patients had received intra-articular steroid injections within 3 months prior to surgery. All patients were diagnosed based on the American College of Rheumatology criteria (22) and evaluated by a certified rheumatologist. Matched healthy cartilage tissues were obtained from traumatic amputees $(n=15$; eight men and seven women, aged 50-79 years) with no history of OA or joint pain. The collected knee OA cartilage samples or healthy cartilage tissues were placed in $0.25 \%$ trypsin-EDTA for digestion, followed by centrifugation at $800 \mathrm{x}$ g for $30 \mathrm{~min}$ at $4^{\circ} \mathrm{C}$ for isolating the chondrocytes. All participants signed informed consent forms and the study was approved by the Ethics Committee of Jingzhou Central Hospital.

Chondrocytes were released from the cartilage samples as previously described (23). Following isolation, the chondrocytes were cultured in Dulbecco's modified Eagle's medium (DMEM, Gibco; Thermo Fisher Scientific, Inc., Waltham, MA, USA) containing 10\% fetal bovine serum (FBS, Gibco; Thermo Fisher Scientific, Inc.), streptomycin (100 mg/ml; Gibco; Thermo Fisher Scientific, Inc.), and penicillin (100 units/ml; Gibco; Thermo Fisher Scientific, Inc.) and maintained in an incubator at $37^{\circ} \mathrm{C}$ with $5 \% \mathrm{CO}_{2}$. During the culture period, the medium was replaced every 2-3 days and cultured chondrocytes at $80 \%$ confluence were used in the experiments.

Cell transfection. The human chondrocytes were transfected with scramble miRNA mimics as the negative control, miR-671-3p mimics, and miR-671-3p inhibitor, respectively (purchased from GenePharma, Shanghai, China). For the rescue
Table I. Clinical characteristics of patients with knee OA and traumatic amputees.

\begin{tabular}{lcc}
\hline Patient characteristic & $\begin{array}{c}\text { Patients with } \\
\text { knee OA }\end{array}$ & $\begin{array}{c}\text { Traumatic } \\
\text { amputees }\end{array}$ \\
\hline Sex & & \\
Male & 9 & 8 \\
Female & 6 & 7 \\
Average age (years) & & \\
$50-59$ & 8 & 6 \\
$60-69$ & 5 & 6 \\
$70-79$ & 2 & 3 \\
Occupation & & 7 \\
Office staff & 5 & 4 \\
Technician & 4 & 1 \\
Housewife & 2 & 3 \\
Other & 4 & \\
\hline
\end{tabular}

OA, osteoarthritis.

experiment, the full-length cDNA sequence for human TRAF3 was obtained from the National Center for Biotechnology Information database, amplified by PCR in OA chondrocytes and cloned into the pcDNA3.1 vector (Invitrogen; Thermo Fisher Scientific, Inc.) to generate the pcDNA3.1-TRAF3 expression vector. The empty pcDNA3.1-vector was used as control. All cell transfections were performed using Lipofectamine 2000 reagent (Invitrogen; Thermo Fisher Scientific, Inc.), following the manufacturer's protocol. At $48 \mathrm{~h}$ post-transfection, the cells were used for the following experiments.

$R N A$ extraction and reverse transcription-quantitative polymerase chain reaction ( $R T-q P C R)$ analysis. Total RNAs from the cartilage tissues and chondrocytes were isolated using the miRNeasy Mini kit and reverse transcribed with the miScript Reverse Transcription kit (Qiagen GmbH, Dusseldorf, Germany) according to the manufacturer's protocol. For RT-qPCR analysis, the TaqMan microRNA Assay kit was used for miR-671-3p and U6 expression analysis. The 20- $\mu 1$ reaction system included $5 \mu \mathrm{l}$ cDNA, $10 \mu \mathrm{l} 2 \mathrm{X}$ SYBR premix ex taq, $0.8 \mu 1$ forward (miR-671-3p, 5'-GCCGCCAAAGTG CTGTTC-3'; U6, 5'-CTCGCTTCGGCAGCACA-3') and reverse (miR-671-3p, 5'-CAGAGCAGGGTCCGAGGTA-3'; U6, 5'-AACGCTTCACGAATTTGCGT-3') primers $(2.5 \mu \mathrm{M})$ and $4.2 \mu \mathrm{ldd} \mathrm{d}_{2} \mathrm{O}$. All reactions were performed in triplicate on a 7900 Real-time system (Applied Biosystems; Thermo Fisher Scientific, Inc.) with the following conditions: $95^{\circ} \mathrm{C}$ for $10 \mathrm{~min}$, followed by 40 cycles at $95^{\circ} \mathrm{C}$ for $15 \mathrm{sec}$, and $60^{\circ} \mathrm{C}$ for $1 \mathrm{~min}$. The expression of U6 was used as endogenous control to normalize the expression level of miR-671-3p. Relative gene expression was calculated using the $2^{-\Delta \Delta \mathrm{Cq}}$ method (24).

ELISA. The synovial fluid and cell supernatants were collected from the 24-well plates. The concentrations of inflammatory cytokines, including interleukin (IL)-1 $\beta$, IL-6, IL-8 and 
TNF- $\alpha$, were detected using ELISA kits (R\&D Systems, Inc., Minneapolis, MN, USA) according to the manufacturer's protocol.

Cell Counting Kit-8 (CCK-8). The chondrocytes were seeded in 96-well plates at the density of 1,000 cells per well. Following transfection, the cells were cultured for another $24,48,72$ or $96 \mathrm{~h}$, respectively. The supernatant was removed, and $10 \mu \mathrm{l}$ of CCK-8 reagent (Beyotime Institute of Biotechnology, Shanghai, China) was added to each well to continue culture for $2 \mathrm{~h}$ at $37^{\circ} \mathrm{C}$. The optical density (OD) value was obtained at the wavelength of $450 \mathrm{~nm}$ using a microplate reader (Bio-Rad Laboratories, Inc., Hercules, CA, USA).

Western blotting. Total protein was extracted from the frozen cartilage tissues and cultured cells using RIPA lysis buffer and then quantified using the Pierce BCA kit (Thermo Fisher Scientific, Inc.). Subsequently, $30 \mu \mathrm{g}$ of protein lysate was loaded onto a $10 \%$ SDS denatured polyacrylamide gel and then transferred onto a polyvinylidene difluoride membrane (EMD Millipore, Bedford, MA. USA). After $2 \mathrm{~h}$ of blocking with $5 \%$ fat-free milk, the membranes were then incubated with primary antibodies against collagen type II $\alpha 1$ chain (COL2A1; 1:5,000; cat. no. ab34712; Abcam, Cambridge, UK), aggrecan (ACAN; 1:2,000; cat. no. ab36861; Abcam), a disintegrin and metalloproteinase with thrombospondin motifs (ADAMTS)-5 (1:250; cat. no. ab41037; Abcam) and GAPDH (1:5,000; cat. no. 10494-1-AP; Proteintech Group, Inc. Chicago, IL, USA) overnight at $4^{\circ} \mathrm{C}$, followed by incubation with horseradish peroxidase-conjugated secondary antibody (1:5,000; cat. no. sc-2054; Santa Cruz Biotechnology, Inc.) for $2 \mathrm{~h}$ at room temperature. The protein bands were detected using the Supersignal ${ }^{\circledR}$ West Pico kit (Thermo Fisher Scientific, Inc.). Image-Pro Plus 6.0 software (Media Cybernetics, Inc., Rockville, MD, USA) was used to quantify the gray value of the bands.

Flow cytometry. Cell apoptosis was assayed by flow cytometry using Annexin V-APC/7-AAD double staining. Briefly, the cells were washed twice with PBS and then resuspended in binding buffer. The cells were then subjected to Annexin V-APC/7-AAD double staining according to the manufacturer's protocol (KeyGEN Biotech Co., Ltd., Nanjing, China). The stained cells were analyzed using a FACScalibur (BD Biosciences, Franklin Lakes, NJ, USA).

Caspase-3 activity assay. The caspase-3 activity assay was performed using the Caspase Colorimetric Assay kit (KeyGEN Biotech Co., Ltd.). Following transfection of the cells for $48 \mathrm{~h}$, the cells were collected and disrupted in lysis buffer on ice for $20 \mathrm{~min}$. The lysates were then incubated with the caspase substrate at $37^{\circ} \mathrm{C}$ in the reaction buffer for $4 \mathrm{~h}$. The OD value at the wavelength of $405 \mathrm{~nm}$ was detected using a microplate reader (Spectra MAX Plus, Molecular Devices, LLC). The relative caspase-3 activity was assessed with the percentage of OD values in the treatment group over that in the control group.

miRNA target prediction and dual-luciferase reporter assay. The miRNAs targets were predicted using the TargetScan (http://www.targetscan.org/), miRanda (http://www.targetscan. org/) and PicTar (http://pictar.mdc-berlin.de/) tools. Among putative genes predicted by the three algorithms, TRAF3 was indicated as a potential target gene of miR-671-3p.

The wild-type TRAF3-3'-UTR (Wt TRAF3) and mutant TRAF3-3'-UTR (Mut TRAF3) containing the putative binding site of miR-671-3p were constructed and cloned into pmirGLO dual luciferase reporter vectors (Promega Corporation, Madison, WI, USA). For the dual reporter luciferase assay, the reporter vectors containing the Wt TRAF3 or Mut TRAF3 and miR-671-3p mimics or scramble were co-transfected into 293T cells (ATCC, Manassas, VA, USA) using Lipofectamine 2000 (Invitrogen; Thermo Fisher Scientific, Inc.). Following $48 \mathrm{~h}$ of incubation, the luciferase activity was analyzed with the Dual Luciferase Reporter Assay system (Promega Corporation) according to the manufacturer's protocol.

Statistical analysis. All statistical analyses were performed using SPSS 20.0 software (IBM Corp. Armonk, NY, USA), and graphs were generated using GraphPad Prism 6 software (GraphPad Software, Inc., San Diego, CA, USA). Data are expressed as the mean \pm standard deviation of at least three experiments. Statistical significance was evaluated using Student's t-test for two-group comparison and one-way analysis of variance for multiple-group comparison. $\mathrm{P}<0.05$ was considered to indicate a statistically significant difference.

\section{Results}

Expression of miR-671-3p is downregulated in knee OA clinical specimens. RT-qPCR analysis was used to evaluate the expression levels of miR-671-3p in 15 knee OA cartilage tissues and 15 normal cartilage tissues. As shown in Fig. 1A, the expression of miR-671-3p in the knee OA cartilage tissues was significantly lower than that in the normal tissues $(\mathrm{P}<0.001)$. In addition, the concentration of several pro-inflammatory cytokines was determined by ELISA. The results showed increased levels of IL-1 $\beta$ (73.0 \pm 5.6 vs. $35.0 \pm 3.6, \mathrm{P}<0.01)$, IL-6 (126.7 \pm 6.1 vs. $48.7 \pm 9.1, \mathrm{P}<0.001)$, IL-8 (37.3 \pm 5.0 vs. $13.3 \pm 4.2$, $\mathrm{P}<0.01)$ and TNF- $\alpha(151.3 \pm 8.0$ vs. $56.7 \pm 7.0, \mathrm{P}<0.001)$ in the knee OA cartilage tissues compared with the normal cartilage tissues (Fig. 1B).

Upregulation of miR-671-3p promotes matrix synthesis and chondrocyte proliferation. To further confirm the regulation of miR-671-3p in the pathogenesis of OA, its mRNA level was also analyzed in chondrocytes from patients with OA and healthy participants, and it was found that chondrocytes from patients with OA had lower expression of miR-671-3p (Fig. 2A, $\mathrm{P}<0.001$ ), which suggested that miR-671-3p may function as a suppressor of OA. Subsequently, scramble control, miR-671-3p mimics, or miR-671-3p inhibitor was transfected into OA chondrocytes, respectively. As shown in Fig. 2B, the miR-671-3p mimics increased the expression of miR-671-3p $(2.0 \pm 0.1$ vs. $0.5 \pm 0.1, \mathrm{P}<0.001)$ and the miR-671-3p inhibitor decreased the expression of miR-671-3p $(0.13 \pm 0.01$ vs. $2.0 \pm 0.1, \mathrm{P}<0.01)$ in the OA chondrocytes. Using gain-of-function and loss-of-function experiments, the effect of the expression of miR-218-5p on cell proliferation was analyzed using a CCK-8 assay. As 

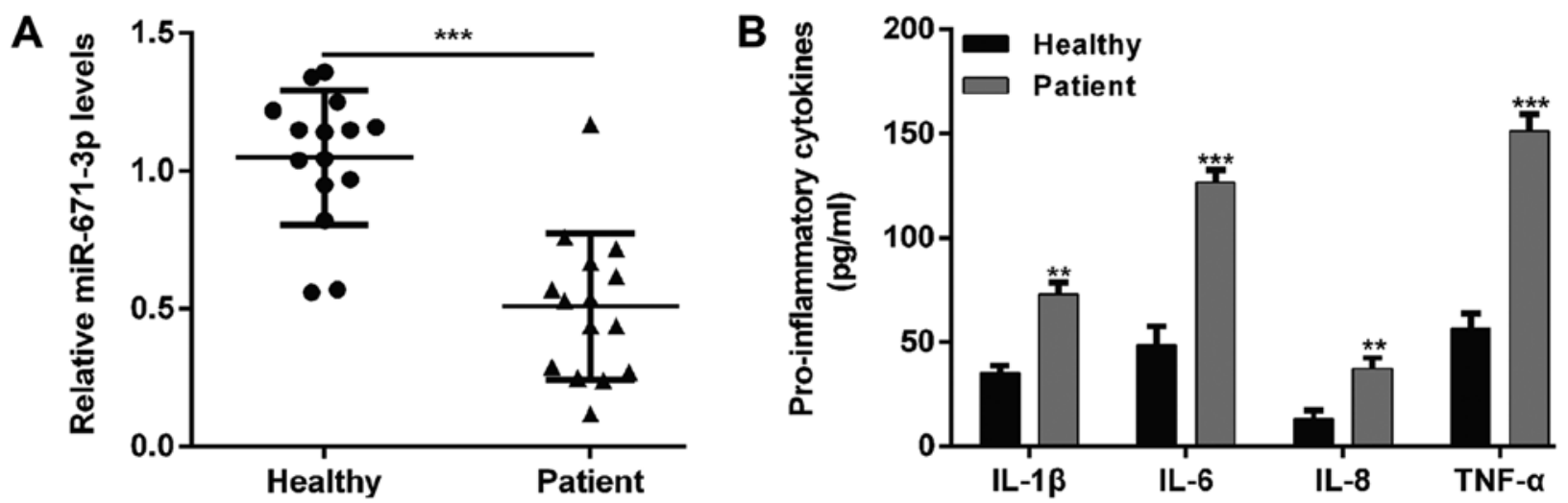

Figure 1. Relative expressions of miR-671-3p and related genes (IL-1 $\beta$, IL-6, IL-10 and TNF- $\alpha$ ) detected in human knee osteoarthritis and normal cartilage tissues. (A) Expression levels of miR-671-3p were detected with reverse transcription-quantitative polymerase chain reaction analysis. (B) An enzyme-linked immunosorbent assay was performed to determine the concentrations of IL-1 $\beta$, IL-6, IL- 8 and TNF- $\alpha$. Data are presented as the mean \pm standard deviation from triplicate experiments. ${ }^{* *} \mathrm{P}<0.01$ and ${ }^{* * *} \mathrm{P}<0.001$, compared with the control healthy group. miR, microRNA; IL, interleukin; TNF, tumor necrosis factor.

A

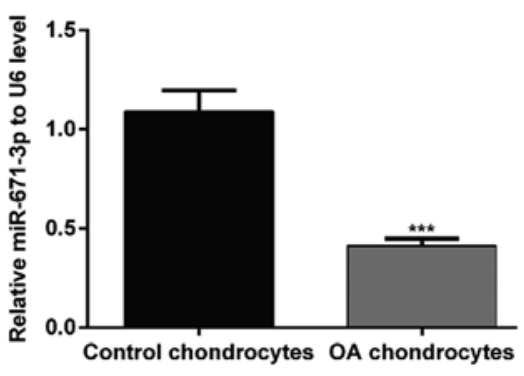

B

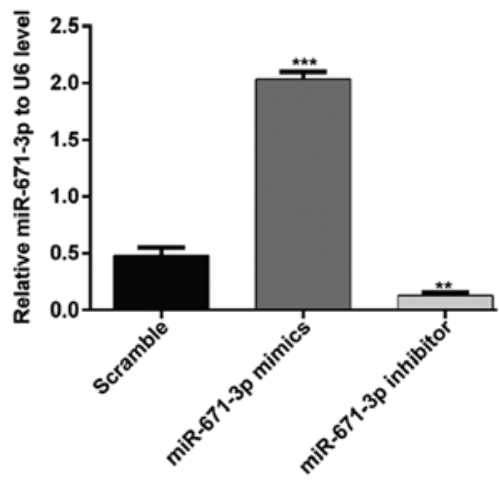

C

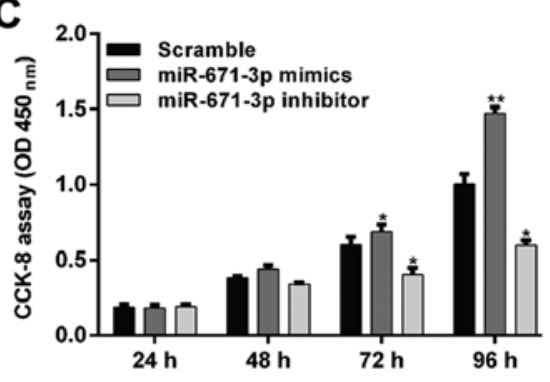

D
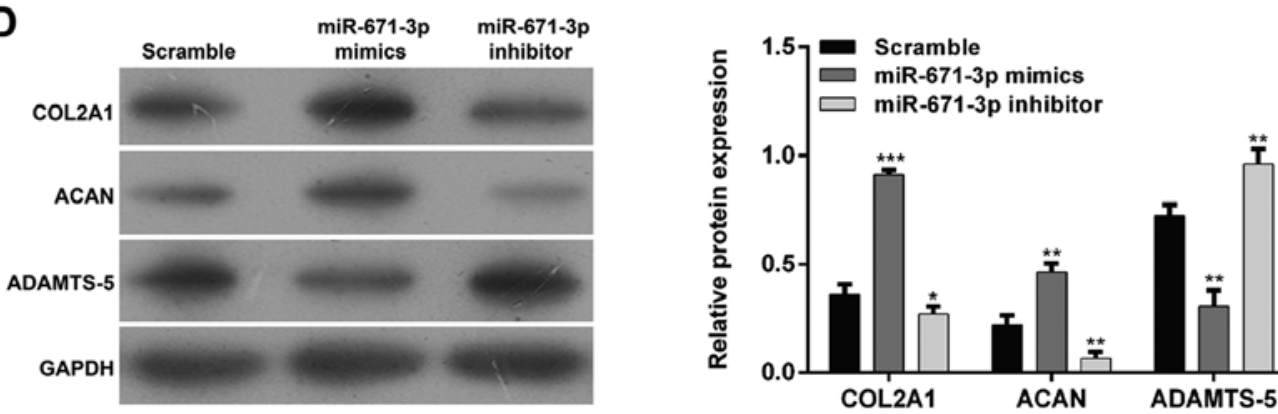

Figure 2. Effects of the expression of miR-671-3p on matrix synthesis and chondrocyte proliferation. (A) Analysis of the expression of miR-671-3p in chondrocytes from healthy participants and patients with OA by RT-qPCR analysis. The miR-control, miR-671-3p mimics or miR-671-3p inhibitor were transfected into human OA chondrocytes. At $48 \mathrm{~h}$ post-transfection, the cells were used for the following experiments. (B) Transfection efficiency of miR-671-3p was analyzed by RT-qPCR analysis. (C) Chondrocyte proliferation was investigated in OA chondrocytes at 24, 48, 72 and $96 \mathrm{~h}$, respectively. Data are presented as the mean \pm standard deviation from triplicate experiments. (D) Expression levels of COL2A1, ACAN and ADAMTS-5 were detected by western blot analysis. ${ }^{*} \mathrm{P}<0.05,{ }^{* *} \mathrm{P}<0.01$ and ${ }^{* * *} \mathrm{P}<0.001$, compared with the scramble group. miR, microRNA; OA, osteoarthritis; RT-qPCR, reverse transcription-quantitative polymerase chain reaction; COL2A1, collagen type II $\alpha 1$ chain; ACAN, aggrecan ADAMTS-5, a disintegrin and metalloproteinase with thrombospondin motifs 5; CCK-8, Cell Counting Kit-8; OD, optical density.

shown in Fig. 2C, the proliferation of chondrocytes was significantly increased when the cells were transfected with miR-671-3p mimics, compared with the scramble group at 72 and $96 \mathrm{~h}(\mathrm{P}<0.05$ and $\mathrm{P}<0.01)$. However, miR-671-3p inhibitor transfection suppressed the proliferation of chondrocytes $(\mathrm{P}<0.05)$. Furthermore, the effect of the expression of miR-671-3p on matrix synthesis biomarkers, including COL2A1, ACAN, and ADAMTS-5, was analyzed in chondrocytes following $48 \mathrm{~h}$ of transfection. The results of the western blotting showed that matrix protein expression
(COL2A1 and ACAN) was increased, whereas the expression of catabolic factor ADAMTS-5 was decreased in chondrocytes transfected with miR-671-3p mimics compared with the scramble control- or miR-671-3p inhibitor-transfected cells (Fig. 2D).

Upregulation of miR-671-3p inhibits chondrocyte apoptosis and inflammation. Accumulating evidence indicates that miRNAs are important in regulating apoptosis and inflammation associated with OA. Therefore, the present study evaluated 
A

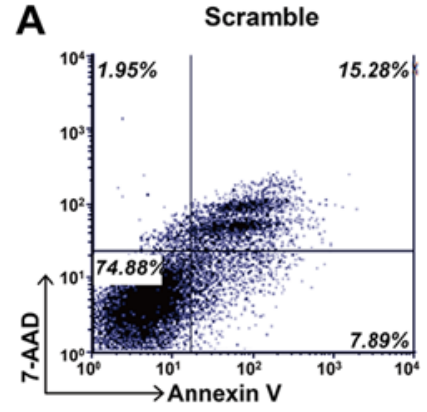

B

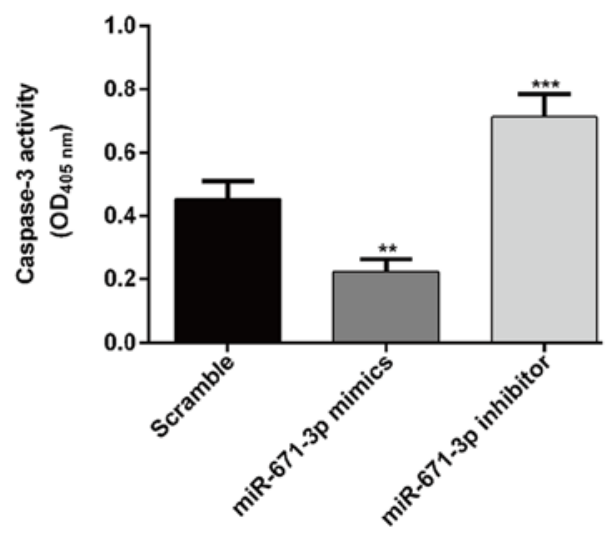

miR-671-3p mimics

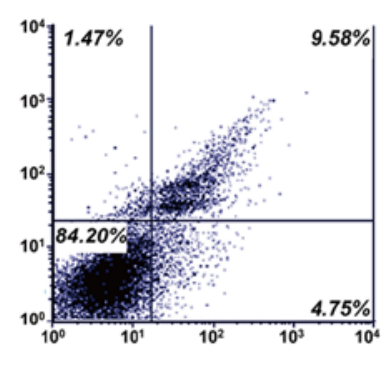

C
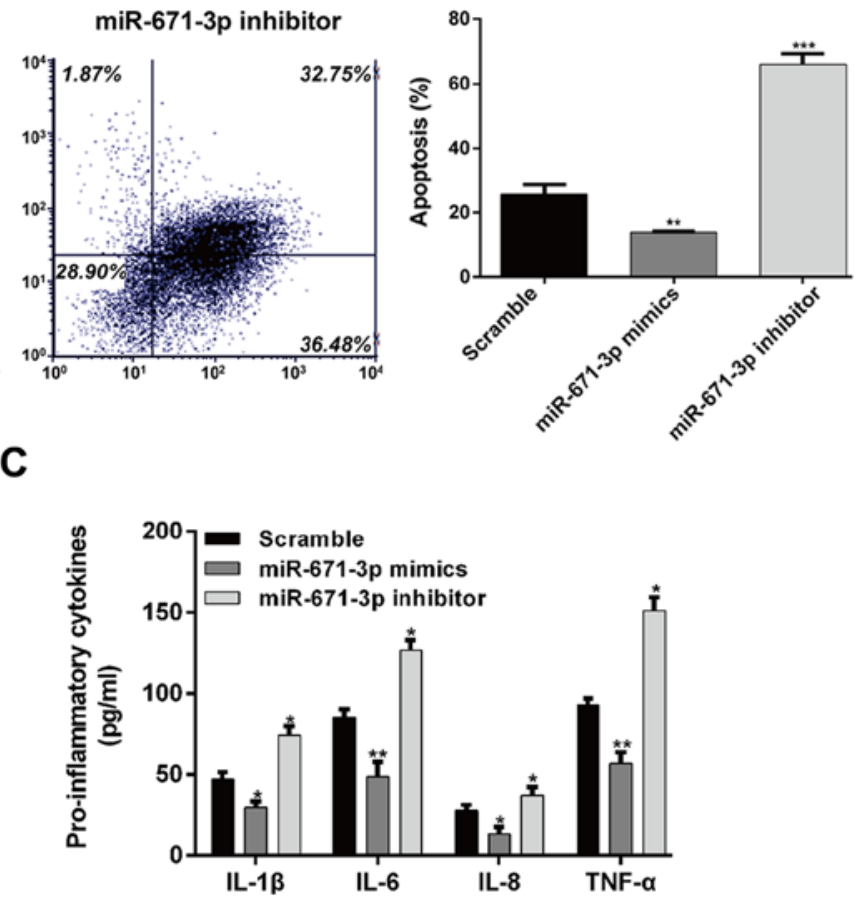

Figure 3. Effects of the expression of miR-671-3p on chondrocyte apoptosis and inflammation. The miR-control, miR-671-3p mimics or inhibitor were transfected into human osteoarthritis chondrocytes, respectively. At $48 \mathrm{~h}$ post-transfection, the cells were used for the following experiments. (A) Apoptotic ability of chondrocytes at $48 \mathrm{~h}$ post-transfection was detected by the flow cytometric analysis. (B) Cell apoptosis was detected using a caspase-3 activity assay. (C) Production of IL-1 $\beta$, IL-6, IL- 8 and TNF- $\alpha$ in the cell culture supernatants was detected using an enzyme-linked immunosorbent assay. Data are presented as the mean \pm standard deviation from triplicate experiments. ${ }^{*} \mathrm{P}<0.05,{ }^{* *} \mathrm{P}<0.01$ and ${ }^{* * *} \mathrm{P}<0.001$, compared with the scramble group. miR, microRNA; IL, interleukin; TNF, tumor necrosis factor; OD, optical density.

whether the altered expression of miR-671-3p affected chondrocyte apoptosis and inflammation. As shown in Fig. 3A, the results of the flow cytometric analysis revealed that the apoptotic rates of the chondrocytes transfected with miR-671-3p mimics and scramble control were $14.0 \pm 0.4$ and $25.7 \pm 2.9 \%$, respectively, with a significant difference $(\mathrm{P}<0.01)$. However, the apoptotic rate in the miR-671-3p inhibitor group was $66.0 \pm 3.4 \%$, which was significantly higher than that in the scramble group $(\mathrm{P}<0.001)$. In addition, the caspase- 3 activity of chondrocytes was significantly suppressed by miR-671-3p mimics transfection, but enhanced by miR-671-3p inhibitor transfection when compared with the scramble group (Fig. 3B, $\mathrm{P}<0.01$ and $\mathrm{P}<0.001$, respectively). Additionally, the pro-inflammatory cytokines IL-1 $\beta$, IL-6, IL- 8 and TNF- $\alpha$ were all significantly decreased by the overexpression of miR-671-3p (Fig. 3C, $\mathrm{P}<0.05$ and $\mathrm{P}<0.01$ ). These results demonstrated that the overexpression of miR-671-3p alleviated cell apoptosis and pro-inflammatory cytokine production in the OA chondrocytes.

Identification of TRAF3 as a functional target of miR-671-3p. To define the target by which miR-671-3p regulates the pathogenesis of OA, the TargetScan, miRanda, PicTar databases were used. The results predicted that TRAF3 was a target of miR-671-3p, which contains a putative target seed sequence (Fig. 4A). Subsequently, firefly luciferase reporters containing the Wt 3'-UTR of TRAF3 or Mut 3'-UTR of TRAF3 were constructed and the luciferase reporter assay was performed. As shown in Fig. 4B, miR-671-3p mimics transfection significantly inhibited the luciferase expression of the reporter containing Wt TRAF3, but not the Mut reporter gene $(\mathrm{P}<0.01)$. In addition, western blot analysis revealed that the upregulation of miR-671-3p significantly reduced the protein levels of TRAF3 and the downregulation of miR-671-3p significantly increased the protein levels of TRAF3 in OA chondrocytes (Fig. 4C). These results suggested that miR-671-3p directly targeted TRAF3 and negatively regulated the expression of TRAF3 by binding to its 3'-UTR.

Restoration of TRAF3 markedly abrogates the effect of miR-671-3p in chondrocytes. To validate whether miR-671-3p regulates the pathogenesis of OA through TRAF3, a rescue experiment was performed by restoring the expression of TRAF3. The CCK-8 assay showed that the restoration of TRAF3 significantly abrogated the enhanced effect of miR-671-3p on the proliferation of OA chondrocytes (Fig. 5A, $\mathrm{P}<0.05$ and $\mathrm{P}<0.01$ ). In addition, the overexpression of TRAF3 partially reversed the miR-671-3p-induced upregulation of COL2A1 and ACAN and downregulation of ADAMTS-5 (Fig. 5B). Furthermore, the protective effects of miR-671-3p on OA-induced apoptosis (Fig. $5 \mathrm{C}, \mathrm{P}<0.01$ and $\mathrm{P}<0.001$ ) and inflammation (Fig. 5D, $\mathrm{P}<0.05$ and $\mathrm{P}<0.01$ ) were also eliminated by restoring the expression of TRAF3. These results suggested that miR-671-3p protected chondrocytes from OA-induced injury by inhibiting TRAF3. 
A

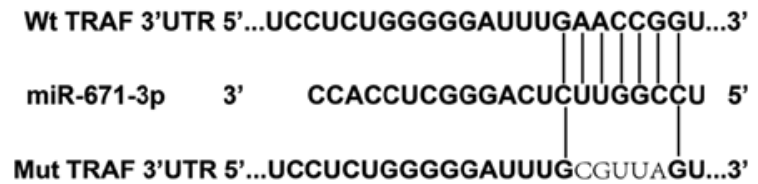

B

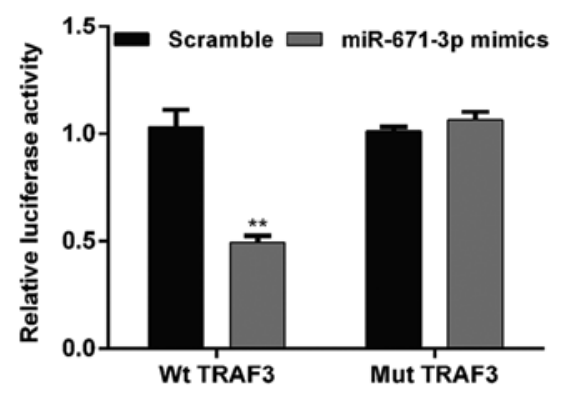

C

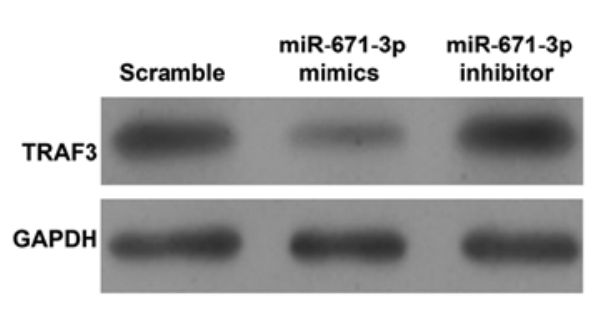

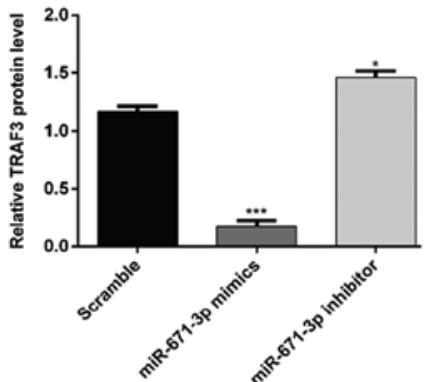

Figure 4. miR-671-3p directly targets TRAF3. (A) Sketch map of the miR-671-3p binding site in the TRAF3 3'-UTR. (B) A 3'-UTR reporter assay was performed in $293 \mathrm{~T}$ cells $48 \mathrm{~h}$ post-transfection. Data are presented as the mean \pm standard deviation from triplicate experiments. (C) Protein expression of TRAF3 was detected in human osteoarthritis chondrocytes following transfection with miR-671-3p mimics or inhibitor using western blot analysis. $\mathrm{P}<0.05$, ${ }^{* *} \mathrm{P}<0.01$ and ${ }^{* * *} \mathrm{P}<0.001$, compared with the scramble group. miR, microRNA; 3'-UTR, 3'-untranslated region; Wt, wild-type; Mut, mutant; TRAF3, tumor necrosis factor receptor-associated factor.

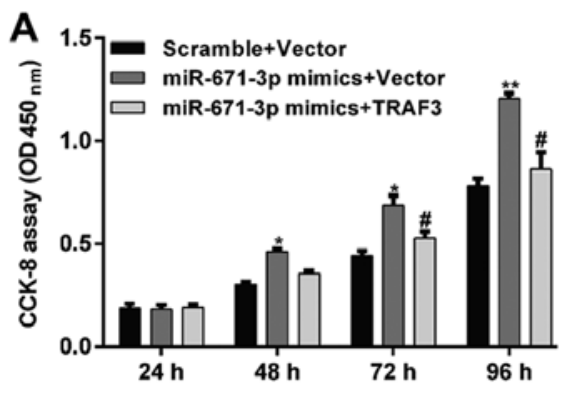

C

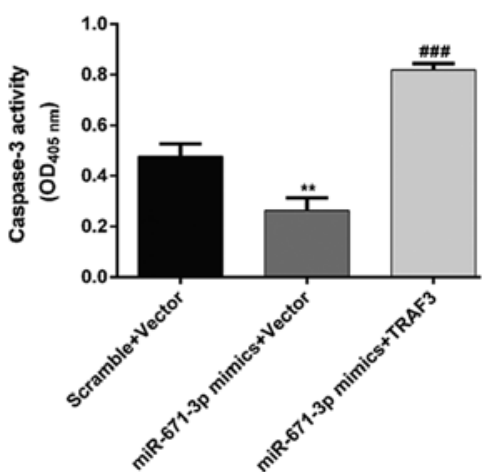

B
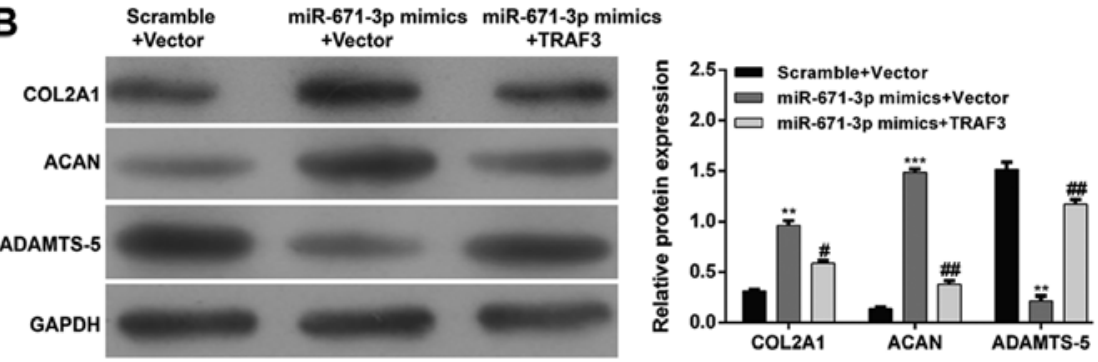

Figure 5. Restoration of TRAF3 markedly abrogates the effect of miR-671-3p. miR-671-3p-overexpressing chondrocytes were transfected with TRAF3, or control vector. (A) Chondrocyte proliferation was investigated in osteoarthritis chondrocytes at 24, 48, 72 and 96 h, respectively. (B) Expression levels of COL2A1, ACAN and ADAMTS-5 were detected by western blot analysis. (C) Cell apoptosis was detected using a caspase-3 activity assay. (D) Production of IL-1 $\beta$, IL-6, IL-8 and TNF- $\alpha$ in the cell culture supernatants was detected by enzyme-linked immunosorbent assay. Data are presented as the mean \pm standard deviation from triplicate experiments. ${ }^{*} \mathrm{P}<0.05,{ }^{* * *} \mathrm{P}<0.01$ and ${ }^{* * *} \mathrm{P}<0.001$, compared with the scramble + vector group; ${ }^{\#} \mathrm{P}<0.05,{ }^{\# \#} \mathrm{P}<0.01$ and ${ }^{\# \# \#} \mathrm{P}<0.001$, compared with the miR-671-3p mimics + vector group. miR, microRNA; TRAF3, tumor necrosis factor receptor-associated factor; COL2A1, collagen type II $\alpha 1$ chain; ACAN, aggrecan ADAMTS-5, a disintegrin and metalloproteinase with thrombospondin motifs 5; CCK-8, Cell Counting Kit-8; OD, optical density.

\section{Discussion}

In recent years, miRNAs have attracted attention due to their association with various human diseases and they provide novel target for the investigation of specific therapeutic agents and intervention tools (25). For example, let-7 exhibits genomic alterations at a high frequency in multiple types of human cancer and is involved in tumor progression and metastasis (26). In addition, Leidinger et al (27) revealed that a 12-miRNA signature may affect 2,354 genes and be involved in nervous system development, including Alzheimer's disease and Parkinson's disease.

The present study investigated whether miR-671-3p expression signatures may be involved in the pathogenesis 
of OA. Substantial effort has been made in understanding the biological function of miRNAs in OA, as reported by Miyaki and Asahara (28). However, there is limited literature reporting the role of miRNAs in the chondrocytes of patients with OA. A study by Yin et al (29) reported that miR-26a and miR-26b contribute to chondrocyte apoptosis in human OA. Zhang et al (30) revealed that the enforced expression of miR-21 depressed the process of chondrogenesis. The results of the present study suggest that the role of miR-671-3p is critical in the pathogenesis of OA. The expression of miR-671-3p was markedly reduced in human knee OA tissues and chondrocytes. Using TargetScan, PicTar and a luciferase reporter assay, TRAF3 was confirmed as a target of miR-671-3p. The overexpression of miR-671-3p promoted chondrocyte survival and proliferation, and inhibited the synthesis of pro-inflammatory cytokines though downregulating TRAF3.

As a complex and dynamic meshwork of proteins, extracellular matrix (ECM) is an important component of multicellular organisms that provides mechanical functions for the orchestration of cellular and tissue organization and function (31). Cartilage in which the catabolism of ECM prevails over its anabolism leads to the development and progression of OA (32). Inflammation is known to be associated with the risk of cartilage loss and progression, and with the clinical characteristics of OA (4). Pro-inflammatory cytokines are considered to be catabolic factors and to control the degeneration of articular cartilage matrix, which indicates their potential as therapeutic targets (33). In the present study, miR-671-3p mimics inhibited the production of pro-inflammatory cytokines IL-1 $\beta$, IL-6, IL-8 and TNF- $\alpha$. In addition, the overexpression of miR-671-3p enhanced matrix protein expression (COL2A1 and ACAN) and suppressed the levels of catabolic factor ADAMTS-5, suggesting that miR-671-3p reduced ECM catabolism and inflammation and increased ECM production, thus being involved in the inhibition of OA development.

TRAF3 is an essential signaling adaptor downstream of multiple TNFR and TLR pathways (34). Pathogen-associated molecular pattern-activated TLRs have been shown to induce the secretion of interferons, pro- and anti-inflammatory cytokines (19). Xiao et al (35) revealed that that the degradation of TRAF3 appeared to be responsible for the promotion of microglia-mediated central nervous system inflammation that was induced by Peli1. In a previous study, TRAF3 was shown to be expressed in myeloid cells and function to attenuate inflammation and tumor progression in mice (36). In the present study, TRAF3 was identified as a direct target of miR-671-3p, and the results further showed that the expression level of TRAF3 was inversely correlated with that of miR-671-3p in chondrocytes. The restoration of TRAF3 significantly abrogated the protective effect of miR-671-3p on cell proliferation, caspase- 3 mediated apoptosis, matrix production and inflammation. These results demonstrated that miR-671-3p is pivotal in the pathogenesis of OA though directly targeting TRAF3. Further investigations are required to further examine the molecular mechanism of miR-671-3p in OA. There were several limitations to the present study, as follows: i) Relatively small sample size; ii) no in vivo animal experiments; iii) additional miRNA transfection methods require validation for transfection of miRNA mimics with strict concentration control (37); iv) mRNA expression levels of the ACAN, COL2A1 and ADAMTS-5 transcripts require determination if conditions permit; v) collection of additional tissue samples is required to confirm the expression levels of ILs in vitro correlated with the patients' samples.

In conclusion, the present study revealed that miR-671-3p was downregulated in OA tissues and chondrocytes. The overexpression of miR-671-3p promoted cell proliferation and suppressed cell apoptosis and inflammation by targeting TRAF3 in OA chondrocytes. These results may provide clues for examining novel therapeutic strategies for preventing inflammation and cartilage destruction in OA.

\section{Acknowledgements}

Not applicable.

\section{Funding}

No funding was received.

\section{Availability of data and materials}

The datasets used and/or analyzed during the present study are available from the corresponding author on reasonable request.

\section{Authors' contributions}

HP made substantial contributions to study conception and design. ZJL performed the experiments. SGC made substantial contributions to acquisition of data. YZY and SJL were involved in collection and analysis of the data XMZ was involved in drafting and revising the manuscript critically for important intellectual content. BH checked the accuracy and integrity of the work and ensured that questions related to the accuracy or integrity of any part of the work are appropriately investigated and resolved.. All authors read and approved the final manuscript.

\section{Ethics approval and consent to participate}

All participants signed informed consent forms and the study was approved by the Ethics Committee of Jingzhou Central Hospital.

\section{Patient consent for publication}

Not applicable.

\section{Competing interests}

The authors declare that they have no competing interests.

\section{References}

1. Johnson VL and Hunter DJ: The epidemiology of osteoarthritis. Best Pract Res Clin Rheumatol 28: 5-15, 2014.

2. Kiadaliri AA, Lohmander LS, Moradi-Lakeh M, Petersson IF and Englund M: High and rising burden of hip and knee osteoarthritis in the Nordic region, 1990-2015. Acta Orthop 89: 177-183, 2018.

3. Showery JE, Kusnezov NA, Dunn JC, Bader JO, Belmont PJ Jr and Waterman BR: The rising incidence of degenerative and posttraumatic osteoarthritis of the knee in the United States Military. J Arthroplasty 31: 2108-2114, 2016. 
4. Kapoor M, Martel-Pelletier J, Lajeunesse D, Pelletier JP and Fahmi H: Role of proinflammatory cytokines in the pathophysiology of osteoarthritis. Nat Rev Rheumatol 7: 33-42, 2011.

5. van der Kraan PM, Blaney Davidson EN, Blom A and van den Berg WB: TGF-beta signaling in chondrocyte terminal differentiation and osteoarthritis: Modulation and integration of signaling pathways through receptor-Smads. Osteoarthritis Cartilage 17: 1539-1545, 2009.

6. Martin JA and Buckwalter JA: Aging, articular cartilage chondrocyte senescence and osteoarthritis. Biogerontology 3: 257-264, 2002

7. Hashimoto S, Ochs RL, Komiya S and Lotz M: Linkage of chondrocyte apoptosis and cartilage degradation in human osteoarthritis. Arthritis Rheum 41: 1632-1638, 1998.

8. Ha M and Kim VN: Regulation of microRNA biogenesis. Nat Rev Mol Cell Biol 15: 509-524, 2014.

9. Tüfekci KU, Meuwissen RL and Genç S: The role of microRNAs in biological processes. Methods Mol Biol 1107: 15-31, 2014

10. Van Peer G, Mets E, Claeys S, De Punt I, Lefever S, Ongenaert M Rondou P, Speleman F, Mestdagh P and Vandesompele J: A high-throughput 3'UTR reporter screening identifies microRNA interactomes of cancer genes. PLoS One 13: e0194017, 2018.

11. Romaine SP, Tomaszewski M, Condorelli G and Samani NJ: MicroRNAs in cardiovascular disease: An introduction for clinicians. Heart 101: 921-928, 2015.

12. Vicente R, Noël D, Pers YM, Apparailly F and Jorgensen C: Deregulation and therapeutic potential of microRNAs in arthritic diseases. Nat Rev Rheumatol 12: 211-220, 2016.

13. Nugent M: MicroRNAs: Exploring new horizons in osteoarthritis. Osteoarthritis Cartilage 24: 573-580, 2016.

14. Akhtar N, Rasheed Z, Ramamurthy S, Anbazhagan AN, Voss FR and Haqqi TM: MicroRNA-27b regulates the expression of matrix metalloproteinase 13 in human osteoarthritis chondrocytes. Arthritis Rheum 62: 1361-1371, 2010.

15. Abouheif $M M$, Nakasa $T$, Shibuya $H$, Niimoto $T$, Kongcharoensombat W and Ochi M: Silencing microRNA-34a inhibits chondrocyte apoptosis in a rat osteoarthritis model in vitro. Rheumatology (Oxford) 49: 2054-2060, 2010.

16. Tardif G, Hum D, Pelletier JP, Duval N and Martel-Pelletier J: Regulation of the IGFBP- 5 and MMP-13 genes by the microRNAs miR-140 and miR-27a in human osteoarthritic chondrocytes. BMC Musculoskelet Disord 10: 148, 2009.

17. Le LT, Swingler TE and Clark IM: Review: The role of microRNAs in osteoarthritis and chondrogenesis. Arthritis Rheum 65: 1963-1974, 2013.

18. Ntoumou E, Tzetis M, Braoudaki M, Lambrou G, Poulou M, Malizos K, Stefanou N, Anastasopoulou L and Tsezou A: Serum microRNA array analysis identifies miR-140-3p, miR-33b-3p and miR-671-3p as potential osteoarthritis biomarkers involved in metabolic processes. Clin Epigenetics 9: 127, 2017.

19. Häcker H, Redecke V, Blagoev B, Kratchmarova I, Hsu LC, Wang GG, Kamps MP, Raz E, Wagner H, Häcker G, et al: Specificity in Toll-like receptor signalling through distinct effector functions of TRAF3 and TRAF6. Nature 439: 204-207, 2006.

20. Vallabhapurapu S, Matsuzawa A, Zhang W, Tseng PH, Keats JJ, Wang H, Vignali DA, Bergsagel PL and Karin M: Nonredundant and complementary functions of TRAF2 and TRAF3 in a ubiquitination cascade that activates NIK-dependent alternative NF-kappaB signaling. Nat Immunol 9: 1364-1370, 2008.

21. Kong QZ, Guo LT, Yang JN, Wang YF, Zhao JX, Kong SH, Zhang M, Yan S and Jin Y: Anti-Inflammatory effects of TRAF-interacting protein in rheumatoid arthritis fibroblast-like synoviocytes. Mediators Inflamm 2016: 3906108, 2016.
22. Altman R, Alarcón G, Appelrouth D, Bloch D, Borenstein D, Brandt K, Brown C, Cooke TD, Daniel W, Feldman D, et al: The American College of Rheumatology criteria for the classification and reporting of osteoarthritis of the hip. Arthritis Rheum 34: 505-514, 1991.

23. Hautier A, Salentey V, Aubert-Foucher E, Bougault C, Beauchef $\mathrm{G}$, Ronzière MC, De Sobarnitsky S, Paumier A, Galéra P, Piperno M, et al: Bone morphogenetic protein-2 stimulates chondrogenic expression in human nasal chondrocytes expanded in vitro. Growth Factors 26: 201-211, 2008.

24. Livak KJ and Schmittgen TD: Analysis of relative gene expression data using real-time quantitative PCR and the 2(-Delta Delta $\mathrm{C}(\mathrm{T})$ ) method. Methods 25: 402-408, 2001

25. Kota J, Chivukula RR, O'Donnell KA, Wentzel EA Montgomery CL, Hwang HW, Chang TC, Vivekanandan P, Torbenson M, Clark KR, et al: Therapeutic microRNA delivery suppresses tumorigenesis in a murine liver cancer model. Cell 137: 1005-1017, 2009.

26. Helland $\AA$, Anglesio MS, George J, Cowin PA, Johnstone CN, House CM, Sheppard KE, Etemadmoghadam D, Melnyk N, Rustgi AK, et al: Deregulation of MYCN, LIN28B and LET7 in a molecular subtype of aggressive high-grade serous ovarian cancers. PLoS One 6: e18064, 2011.

27. Leidinger P, Backes C, Deutscher S, Schmitt K, Mueller SC, Frese K, Haas J, Ruprecht K, Paul F, Stähler C, et al: A blood based 12-miRNA signature of Alzheimer disease patients. Genome Biol 14: R78, 2013.

28. Miyaki S and Asahara $\mathrm{H}$ : Macro view of microRNA function in osteoarthritis. Nat Rev Rheumatol 8: 543-552, 2012.

29. Yin X, Wang JQ and Yan SY: Reduced miR26a and miR26b expression contributes to the pathogenesis of osteoarthritis via the promotion of $\mathrm{p} 65$ translocation. Mol Med Rep 15: 551-558, 2017.

30. Zhang Y, Jia J, Yang S, Liu X, Ye S and Tian H: MicroRNA-21 controls the development of osteoarthritis by targeting GDF-5 in chondrocytes. Exp Mol Med 46: e79, 2014.

31. Naba A, Clauser KR, Ding H, Whittaker CA, Carr SA and Hynes RO: The extracellular matrix: Tools and insights for the 'omics' era. Matrix Biol 49: 10-24, 2016.

32. Rahmati M, Nalesso G, Mobasheri A and Mozafari M: Aging and osteoarthritis: Central role of the extracellular matrix. Ageing Res Rev 40: 20-30, 2017.

33. Goldring MB: Osteoarthritis and cartilage: The role of cytokines. Curr Rheumatol Rep 2: 459-465, 2000.

34. Häcker H, Tseng PH and Karin M: Expanding TRAF function: TRAF3 as a tri-faced immune regulator. Nat Rev Immunol 11: 457-468, 2011.

35. Xiao Y, Jin J, Chang M, Chang JH, Hu H, Zhou X, Brittain GC, Stansberg C, Torkildsen $\varnothing$, Wang X, et al: Pelil promotes microglia-mediated CNS inflammation by regulating Traf3 degradation. Nat Med 19: 595-602, 2013.

36. Lalani AI, Moore CR, Luo C, Kreider BZ, Liu Y, Morse HC III and Xie P: Myeloid cell TRAF3 regulates immune responses and inhibits inflammation and tumor development in mice. J Immunol 194: 334-348, 2015

37. Jin HY, Gonzalez-Martin A, Miletic AV, Lai M, Knight S, Sabouri-Ghomi M, Head SR, Macauley MS, Rickert RC and Xiao C: Transfection of microRNA mimics should be used with caution. Front Genet 6: 340, 2015. 